OPEN ACCESS

Approved by:

Frontiers Editorial Office,

Frontiers Media SA, Switzerland

*Correspondence:

Fan Lu

lufan@eye.ac.cn

Jiawei Zhou

zhoujw@eye.ac.cn

${ }^{\dagger}$ Co-first authors

Received: 22 May 2019 Accepted: 24 May 2019

Published: 12 June 2019

Citation:

Wang $Y$, He Z, Liang Y, Chen $Y$, Gong L, Mao Y, Chen X, Yao Z, Spiegel DP, Qu J, Lu F, Zhou J and Hess RF (2019) Corrigendum: The Binocular Balance at High Spatial Frequencies as Revealed by the Binocular Orientation Combination Task. Front. Hum. Neurosci. 13:196. doi: 10.3389/fnhum.2019.00196

\section{Corrigendum: The Binocular Balance at High Spatial Frequencies as Revealed by the Binocular Orientation Combination Task}

Yonghua Wang ${ }^{1,2,3 t}$, Zhifen He $\mathrm{He}^{2,3 \dagger}$, Yunjie Liang ${ }^{2,3+}$, Yiya Chen ${ }^{2,3}$, Ling Gong ${ }^{2,3}$, Yu Mao ${ }^{2,3}$, Xiaoxin Chen ${ }^{2,3}$, Zhimo Yao ${ }^{2,3}$, Daniel P. Spiegel ${ }^{4}$, Jia Qu ${ }^{2,3}$, Fan Lu ${ }^{2,3 *}$, Jiawei Zhou ${ }^{2,3 *}$ and Robert F. Hess ${ }^{5}$

${ }^{1}$ The First Affiliated Hospital of Wenzhou Medical University, Wenzhou, China, ${ }^{2}$ School of Ophthalmology and Optometry and Eye Hospital, Wenzhou Medical University, Wenzhou, China, ${ }^{3}$ State Key Laboratory of Ophthalmology, Optometry and Vision Science, Wenzhou Medical University, Wenzhou, China, ${ }^{4}$ Vision Sciences, Essilor R\&D, Center for Innovation and Technology, Singapore, Singapore, ${ }^{5}$ McGill Vision Research, Department of Ophthalmology, McGill University, Montreal, QC, Canada

Keywords: binocular eye dominance, spatial frequency, binocular orientation combination, binocular phase combination, contrast-gain

\section{A Corrigendum on}

The Binocular Balance at High Spatial Frequencies as Revealed by the Binocular Orientation Combination Task

by Wang, Y., He, Z., Liang, Y., Chen, Y., Gong, L., Mao, Y., et al. (2019). Front. Hum. Neurosci. 13:106. doi: 10.3389/fnhum.2019.00106

An author name was incorrectly spelled as "Zhifeng He." The correct spelling is "Zhifen He."

The authors apologize for this error and state that this does not change the scientific conclusions of the article in any way. The original article has been updated.

Copyright ( 2019 Wang, He, Liang, Chen, Gong, Mao, Chen, Yao, Spiegel, Qu, Lu, Zhou and Hess. This is an open-access article distributed under the terms of the Creative Commons Attribution License (CC BY). The use, distribution or reproduction in other forums is permitted, provided the original author(s) and the copyright owner(s) are credited and that the original publication in this journal is cited, in accordance with accepted academic practice. No use, distribution or reproduction is permitted which does not comply with these terms. 